\title{
Making a Case for use of Digital Interventions to Manage Adolescent Perinatal Depression in Nigeria
}

\author{
Lola Kola*1,2 \\ ${ }^{1}$ Department of Psychiatry, University of Ibadan, Nigeria \\ ${ }^{2}$ Department of Sociology and Psychology, Lead City University, Ibadan
}

*Corresponding author: Lola Kola, WHO Collaborating Centre for Research and Training in Mental Health, Neurosciences and Drug and Alcohol abuse, Department of Psychiatry, College of Medicine, University of Ibadan, Nigeria.

To Cite This Article: Lola Kola, Making a Case for use of Digital Interventions to Treat Adolescent Perinatal Depression in Nigeria. Am J Biomed Sci \& Res. 2019 - 6(4). AJBSR.MS.ID.001054. DOI: 10.34297/AJBSR.2019.06.001054.

Received: 門 November 27, 2019; Published: 門 December 04, 2019

\section{Opinion}

Nigeria has a fast-growing young population, and almost $43 \%$ of her 200 million people are aged between 0 and 14 years. The national fertility rate for girls age 15-19 years is 122 per thousand birth [1] and like much of the region, adolescent pregnancy is a pressing public health issue in the country. In general, adolescents face several psychological, social and financial challenges in the perinatal period irrespective of if their pregnancies are planned, or unplanned. As such, perinatal depression is higher in this age group than among older pregnant women. African countries have a large treatment gap for mental disorders. In Nigeria due to insufficient manpower, maternal and child health care services in primary care is delivered by non-specialists through a process of task shifting [2]. The World Health Organization (WHO) Mental Health Gap Action Plan program is one of the evidenced-based care initiatives to support the delivery of care for common mental disorders in low resource settings through task shifting. The mhGAP-IG has been adapted to the Nigerian context and adopted for use in primary care by the Nigerian National Council of health in August 2013. However despite the effectiveness of psychosocial interventions when delivered by non-specialists in the treatment of perinatal depression, adolescence distinct developmental related challenges, self-stigma (shame relating to getting pregnant at a young age), public stigma (from health care providers and others in the community) are factors that often limit the use of available health care facilities by perinatal adolescents. Effective, flexible, and convenient to access Interventions are urgently needed to address the health care needs of pregnant adolescents to reduce mental health morbidity in this population.

Digital Health including the use of mobile phones and other wireless devices in healthcare, (mHealth) is increasingly being adopted for service delivery in the field of behavioral and mental health. Its potentials to revolutionize healthcare in low resource settings by making it more efficient and patient-centered has been acknowledged in many of such settings [3]. mHealth, the most commonly form of digital intervention used in mental health, reduces the complexities of accessing healthcare by taking care delivery outside the confines of the hospital walls, thereby giving patients the flexibility and privacy of access at their convenience. When combined with face to face care in a 'Blended care' approach, mobile phones have shown strong potentials of effectiveness in improving patients' outcomes by increasing contact with care.

Nigeria is one of the three countries in the African region with the fastest-growing number of mobile phone users. The smartphone infrastructure in Africa is far more developed than roads and water of the region [4]. The availability of affordable mobile technologies in low-income settings have enhanced their potentials to move clinical research forward [5]. The impetus to scaling up existing evidence-based intervention for perinatal depression among adolescents in low resource settings calls for effective, flexible, and convenient to access interventions in addressing the health care needs of young mothers. There are needs for pragmatic innovative approaches to make mental health care available and convenient to access for young mothers both in urban and rural Africa. The ubiquity of mobile phones in the African region presents a context where innovative mobile health (mHealth) approaches can be developed to address a major public health concern like adolescent perinatal depression. Mobile health interventions are practical ways to deliver intervention to youths [6]. The fact that many young people are often attached to their mobile phones makes delivery of care through this avenue a practical approach to keep them engaged with care. Health care delivery to adolescents' mothers through 
mobile phones as adjunct treatment to face to face care delivered in the clinics, presents a practical way of scaling mental health care services in this population through the increasing of their contact with health care.

I am optimistic about the opportunities mobile phones present in scaling up care for perinatal adolescents in Nigeria. mHealth presents different simple and effective ways to deliver safe care to adolescent mothers who have difficulties going to the clinics for various reasons. Basic mobile phone functions such as phone to phone calls, clinician or computer-generated SMS messages can be used to deliver health information to young mothers to improve their treatment outcomes [7]. Follow-up calls from clinicians can be made to patients at a preplanned time (to ensure privacy and availability), and SMS messages can also be read at the patient's convenience. Smartphones (mobile phone application) with preloaded management components of the mhGAP-IG can be utilized by perinatal women as a self-management guide. Short audiovisual contents can be used to increase user engagement and to overcome literacy barriers among women of low education [8]. The possibility of access to basic contents and functionality in offline modes makes it possible for mobile phone application to be used by perinatal women in areas with poor internet connectivity. Notifications could also serve as follow up and alternative of "interaction with health workers" in a less intrusive way and as reminders of necessary activities such as promotion of physical activities and child immunization.
The opportunities to explore are enormous. This is an urgent call for a paradigm shift from sole reliance on face to face care delivery approaches for mental health care in Africa, to pragmatic technology-assisted innovation particularly among youths. The technological revolution in sub-Saharan Africa is well suited to digitally supported mental healthcare delivery to support perinatal adolescents.

\section{References}

1. Cortez R, Saadat S, Marinda E, Oluwole O (2015) Adolescent Sexual and Reproductive Health in Nigeria: Health, nutrition, and population global practice knowledge brief. Washington, USA.

2. Kakuma, Ritsuko, Harry Minas, Nadja Van Ginneken, Mario R Dal Poz, et al. (2011) Human resources for mental health care: current situation and strategies for action. The Lancet 378(9803): 1654-1663.

3. Jacobson LE, Labrique AB (2018) Information Communication Technology and Health. Foundations for Global Health Practice 217.

4. World Bank (2013) Fact Sheet: Infrastructure in Sub-Saharan Africa.

5. Chibanda D (2017) The future of psychiatry in Africa: thinking outside the box. Lancet Psychiatry 4(10): 741-742.

6. Fedele DA, Cushing CC, Fritz A, Amaro CM, Ortega A (2017) Mobile health interventions for improving health outcomes in youth: a meta-analysis. JAMA pediatrics 171(5): 461-469.

7. Perrier T, Dell N, DeRenzi B, Anderson R, Kinuthia J, et al. (2015) Engaging pregnant women in Kenya with a hybrid computer-human SMS communication system. In Proceedings of the $33^{\text {rd }}$ Annual ACM Conference on Human Factors in Computing Systems pp. 1429-1438.

8. Brian RM, Ben-Zeev D (2014) Mobile health (mHealth) for mental health in Asia: objectives, strategies, and limitations. Asian journal 10: 96-100. 